\title{
MOTION ADAPTIVE DE-INTERLACING BY HORIZONTAL MOTION DETECTION AND ENHANCED ELA PROCESSING
}

\author{
Shyh-Feng Lin, Yu-Lin Chang, and Liang-Gee Chen \\ DSP/IC Design Lab, Department of Electrical Engineering \\ National Taiwan University, Taipei, Taiwan, R.O.C.
}

\begin{abstract}
A motion adaptive de-interlacing algorithm and its hardware architecture are presented in this paper. It consists of the directional interpolation - ELA with median processing, and 4-field horizontal motion detection. The edges can be sharper while the ELA with median processing is adopted. Enhanced ELA with median processing can prevent the happening of the bursting pixels. Proposed 4-field horizontal motion detection discovers faster motion and makes more accurate motion detection. The proposed method achieves hardware considerations on low complexity, low memory usage, and high speed processing. The experimental results show that the image quality of the proposed algorithm is superior to previous systems, and the cost is also more efficient. It is very suitable to be integrated into any display device such as projection TV and Plasma display.
\end{abstract}

\section{INTRODUCTION}

De-interlacing is an important process which converts ordinary interlaced TV sequences into progressive ones in order to output on the progressive devices (e.g. CRTs, Plasma Display Panels, TFT-LCDs). Some defects such as edge-flicker, line crawling will cause visual artifacts which let audiences uncomfortable if de-interlacing is not done perfectly.

Two methods of low complexity de-interlacing methods are $\mathrm{BOB}[1]$ and Weave[1] which are adopted into the software DVD Player. BOB is an intra-field de-interlacing method that uses a single field to reconstruct one progressive frame. However, the vertical resolution is halved and the image is blurred. Weave is a simple de-interlacing method directly combining two interlaced fields into one progressive frame. But the line crawling effect would happen in the motion area. A directional interpolation ELA for BOB had been proposed by Kuo, Liao, and Lin [2]. The ELA algorithm enhances the sharpness of the interpolated image.

The motion adaptive de-interlacing integrates the advantages of the intra-field de-interlacing and the inter-field de-interlacing. It detects the motion areas then adopts intra-field de-interlacing into the motion areas and inter-field de-interlacing into the static areas. A same parity 2-field morphological motion detection mechanism has been proposed by Lin, Chang, and Chen [3], but it can not detect very high-speed motion.

Some motion adaptive techniques[4]-[7] had been presented to improve the image quality. Sugiyama and Nakamura[4] proposed a motion adaptive de-interlacing method with motion compensation. They used motion compensated method combining with the adaptive interpolation to reconstruct the missing field. Hilman, Park, and Kim[5] proposed a motion-compensated frame-rate conversion algorithm with interpolation to reduce the $3: 2$ pulldown artifacts. Ville[6] proposed a motion adaptive technique on a fuzzy motion detector. Patti et al.,[7] adopt the global motion compensation into motion adaptive de-interlacing to remove the artifacts due to camera panning or zooming. All those methods can provide good image quality for de-interlacing. However, these methods take a lot of computational power and expense for consumer electronic products.

In this paper, a motion adaptive de-interlacing method with enhanced ELA processing, and 4-field horizontal motion detection is proposed. The overview of intra-field and inter-field deinterlacing will be discussed in the section 2 . The proposed ELA with median processing, 4-field horizontal motion detection will be described in the section 3 . Section 4 states the considerations for hardware implementation, and shows the experimental results. At last, the conclusion remarks the proposed methods.

\section{OVERVIEW}

De-interlacing methods can be roughly divided into three categories - the Intra-field de-interlacing, the Inter-field de-interlacing, and the motion adaptive de-interlacing.

\subsection{Intra-field De-interlacing}

The intra-field de-interlacing means to construct a whole frame just based on original one field. The easiest and the most common method of intra-field de-interlacing is bilinear interpolation. The bilinear interpolation blurs the edge because it is a running average of the neighboring pixels. However, it is the easiest method among all the intra-field methods.

\subsection{Inter-field De-interlacing}

Inter-field de-interlacing finds the best match from the forward field or the backward field to reconstruct a new frame. The video quality will be better than intra-field interpolation, especially in the static area. The best method for inter-field de-interlacing is the motion compensated method.

\subsection{Motion Adaptive De-interlacing}

The most important part of the motion adaptive de-interlacing is to detect where the motion areas are. If the motion detection can be done correctly, the output image quality can be as high as possible.

\section{PROPOSED DE-INTERLACING}

The block diagram of proposed methods is shown in Fig. 1. Three field buffers are used to store the reference data. The 4field horizontal motion detection calculates the difference between the forward-forward field to current field and the forward 


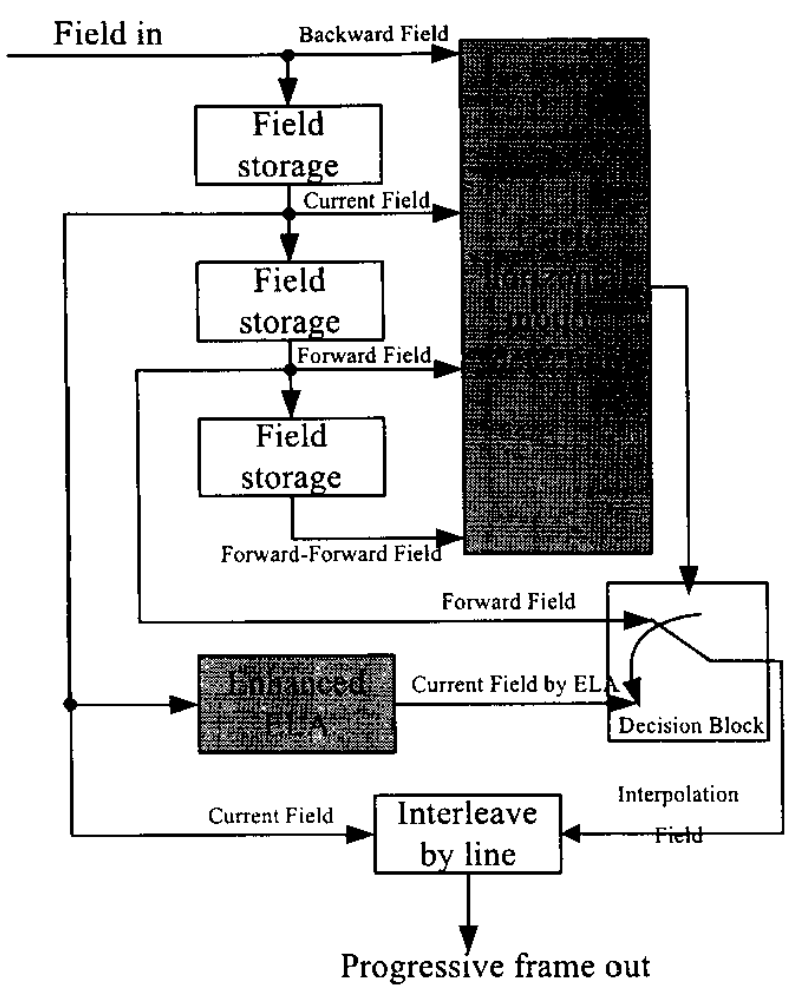

Fig. 1 Block Diagram of the Proposed De-interlacing method

field to backward field then gives motion information to the decision block. The enhanced ELA module does the directional edge interpolation according to the current-field information. Decision block receives the motion information then uses the forward field in the static area, and uses the current-field by ELA in the motion area. At last, the current field and interpolationfield are combined into a progressive frame.

\subsection{ELA with Median Processing}

The ELA algorithm can detect the edges in the original field and generates a new frame. The new field possesses high-contrast on the edge of the image. We enhanced the ELA to detect 5 pixels in the previous and next scan line as shown in Fig. 2. Firstly, five differences are calculated from the five directions. Then doing the interpolation along the direction which has the smallest difference. The median of three pixels - the produced pixel, the corresponding pixels in the previous line and in the next line can prevent the happening of the bursting pixels. Bursting pixels may appear in our motion detection, the ELA with Median processing is designed to reduce the bursting pixels. So ELA with Median processing has better image quality than usual ELA in our alogithm.

\subsection{4-Field Horizontal Motion Detection}

The same parity 2-field motion detection is shown in Fig. 3. The field difference means the motion area of the current field. Pixels detected to be moving will be processed by the intra-field deinterlacing. But as for this case, the motion areas of the current

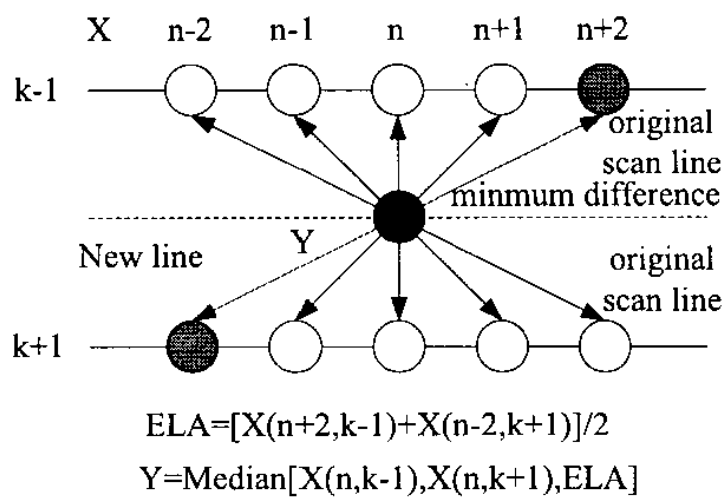

Fig. 2 ELA with median processing

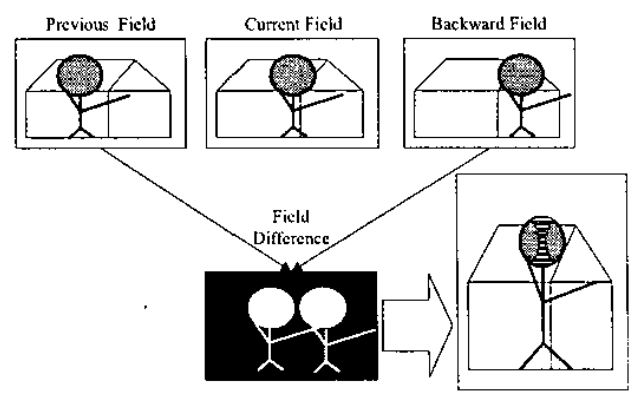

Fig. 3 The 2 field motion detection

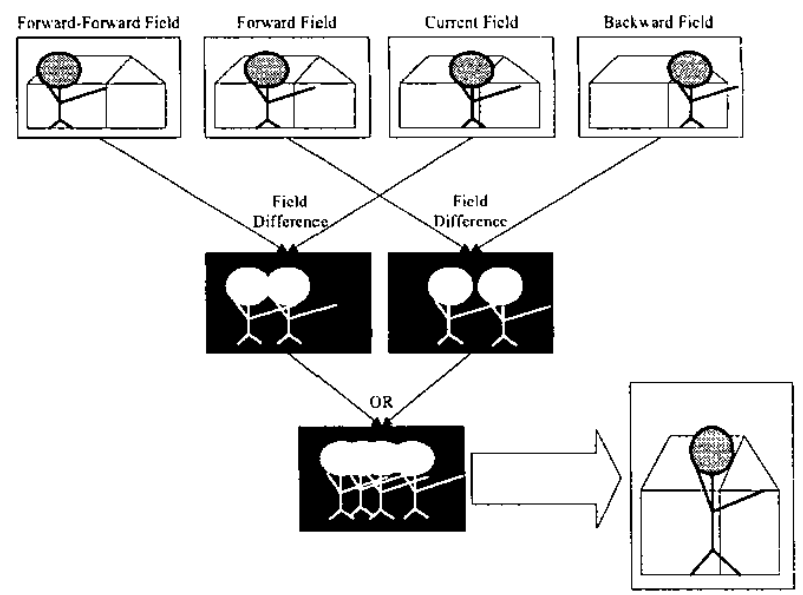

Fig. 4 The 4 field motion detection

field are not correctly detected. The forward field would be filted into the middle of the circle, and the artifact turns up therc.

The 2-field difference would be insufficient for good image quality. The extension of 2-field motion detection is 4 -field motion detection shown in Fig. 4. The difference between forwardforward field and current field are introduced to obtain more motion areas and can solve the artifact which produced by the fast motion.

The severest defect on interlaced sequences displayed on progressive devices is line crawling effect. A temporal prediction 


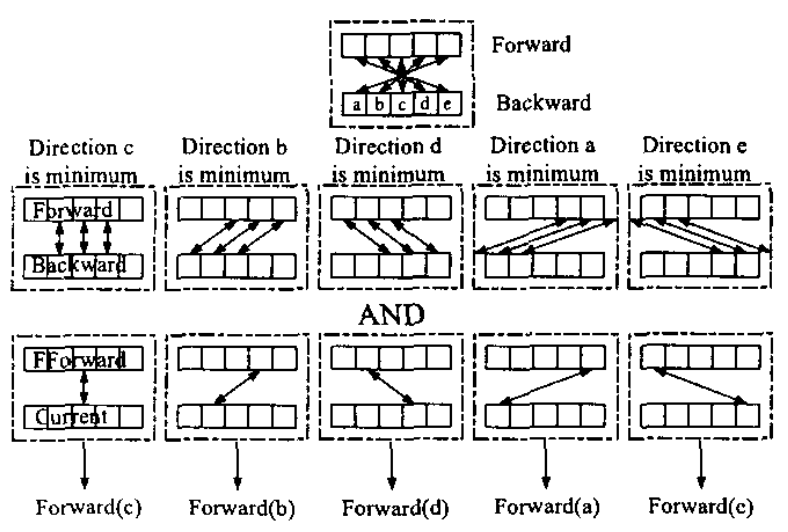

Fig. 5 4-field horizontal motion detection

could help to know where an object is going to move. So our proposed 4-field horizontal motion detection combines the temporal prediction and the 4 -field motion detection and is shown in Fig. 5.

The advantage of the 4-field horizontal motion detection is to remove the detection error by large motion and different parityfield check.

\section{RESULTS}

This section describes the implementation issues and results of proposed de-interlacing methods.

\begin{tabular}{|c|c|c|c|c|c|}
\hline & $\begin{array}{c}\text { Complex- } \\
\text { ity }\end{array}$ & Read & Write & $\begin{array}{c}\text { Mem- } \\
\text { ory } \\
\text { Usage } \\
\text { [bytes] }\end{array}$ & $\begin{array}{c}\text { Instruc- } \\
\text { tion } \\
\text { Counts } \\
\text { [MIPS] }\end{array}$ \\
\hline Bilinear & $\mathrm{O}(\mathrm{n})$ & $1 \mathrm{n}$ & $\ln$ & 1 & 481 \\
\hline ELA & $\mathrm{O}(\mathrm{n})$ & $2 \mathrm{n}$ & $\ln$ & 10 & 879 \\
\hline M.C. & $\mathrm{O}\left(\mathrm{n}^{2} \times \mathrm{SR}^{2}\right)$ & $>10 \mathrm{n}$ & $\ln$ & 528 & 30,248 \\
\hline Proposed & $\mathrm{O}(\mathrm{n})$ & $5 \mathrm{n}$ & $\ln$ & 25 & 1,224 \\
\hline
\end{tabular}

Table 1 The comparison results of complexity, memory access, memory usage, and instruction counts

\begin{tabular}{|c|c|c|c|c|c|r|}
\hline Name & Merged & Bilinear & ELA & $\begin{array}{c}\text { 2 Field + } \\
\text { Morphol- } \\
\text { ogy }\end{array}$ & MC & Proposed \\
\hline Silent & 29.52 & 29.15 & 26.72 & 39.22 & 39.53 & 39.82 \\
\hline Weather & 31.01 & 26.93 & 25.55 & 37.49 & 36.89 & 40.49 \\
\hline Mobile & 17.83 & 24.92 & 23.99 & 25.17 & 25.04 & 25.83 \\
\hline $\begin{array}{c}\text { Mother } \\
\text { Daughter }\end{array}$ & 33.59 & 32.89 & 30.82 & 38.05 & 42.42 & 42.65 \\
\hline Container & 31.01 & 28.14 & 25.04 & 35.33 & 33.97 & 37.81 \\
\hline Stefan & 11.86 & 26.91 & 25.49 & 27.38 & 26.60 & 26.12 \\
\hline Dancer & 23.19 & 36.15 & 33.41 & 32.95 & 36.20 & 34.06 \\
\hline Foreman & 23.30 & 29.96 & 27.41 & 30.83 & 32.18 & 33.21 \\
\hline $\begin{array}{c}\text { Hall Moni- } \\
\text { tor }\end{array}$ & 30.61 & 30.68 & 27.75 & 35.31 & 38.50 & 39.14 \\
\hline $\begin{array}{c}\text { Table Ten- } \\
\text { nis }\end{array}$ & 23.89 & 27.32 & 25.85 & 32.46 & 33.95 & 35.11 \\
\hline Coastguard & 22.59 & 27.84 & 26.32 & 29.00 & 28.13 & 30.45 \\
\hline
\end{tabular}

Table 2. PSNR Comparison (dB)

\subsection{Complexity Comparison}

The comparisons of the complexity, memory access frequency, local buffer usage, and the instruction counts between the methods are described in the table 1 .

The " $n$ " in Table 1 means the pixel numbers to be processed and the "SR" means search range in the motion compensation. The complexity of bilinear interpolation and ELA is O(n) because they are just a kind of FIR filter. There are many kinds of MC de-interlacing method, but their complexities are too high for comparing with motion adaptive methods. So a common $\mathrm{MC}$ deinterlacing method is compared here for reference. The motion compensated de-interlacing needs full-search motion estimation, and the complexity of the full search motion estimation is $\mathrm{O}\left(\mathrm{n}^{2} \mathrm{x}\right.$ $\mathrm{SR}^{2}$ ). The complexity of the motion compensation in the MC deinterlacing is smaller than the motion estimation part, so its complexity is ignored. The complexity of the proposed method is similar to ELA, and the 4 field horizontal motion detection has a complexity of $O(n)$. So the overall complexity of our proposed method is $O(n)$.

Due to the pipeline architecture, the bilinear interpolation should read in one pixel into local buffer while processing each pixel. As for ELA, it needs to examine two pixels on two different scanlines. The memory access frequency of the motion estimation part of the $\mathrm{MC}$ de-interlacing varies in the different designs, but the reading frequency of the memory will be more than 10 times per pixel. Our proposed method read 5 pixels from memory each time while the 4-field horizontal motion detection is utilized.

The instruction counts are profiled by I-prof with gec 3.0.2 and the target video sequence is for CIF 30FPS. There is much to optimize in the $\mathrm{C}$ codes for hardware implementation. But it is shown that the proposed method needs only 1.2GIPS for realtime de-interlacing. The instruction count of the proposed method is similar to those of the intra-field de-interlacing methods and is much smaller than the MC methods.

It is obviously that motion compensated de-interlacing is the most complex and time-wasting method, and the proposed algorithm possesses less memory access frequency than the motion compensated de-interlacing and it has the same complexity with the intra-field de-interlacing.

\subsection{PSNR Comparison}

Several video test sequences have been made interlaced and recovered by the de-interlacing methods. The PSNR comparison is shown in the table 2 . The proposed method achieves the highest PSNR performance in many of the sequences except some sequences with very fast motions, but it can still retain high PSNR in these fast motion sequences.

\subsection{Subject View Comparison}

The sequence "Pendulum" emphasizes on the texture preservation and edge sharpness while de-interlacing is done. The original sequence can divide into three parts. The most important of all is the oscillating pendulum; it swings from the left of the screen to the right back and forth. In the combined frame, the 
line crawling appears at the motion area of the pendulum. The second part is the title "OK", which are constituted of " $\mathrm{O}$ " only in the even fields and " $\mathrm{K}$ " only in the odd fields.

The third part of the sequence is the logo rotating at the rightdown corner of the screen. The rotating logo acts like a non-rigid body, and the real appearance of non-rigid bodies are often lost by $\mathrm{MC}$ de-interlacing. However, motion adaptive de-interlacing methods can reconstruct the non-rigid bodies very well. The original two fields and the directly combined frame are shown in the Fig. 6(a) (b)(c) separately.

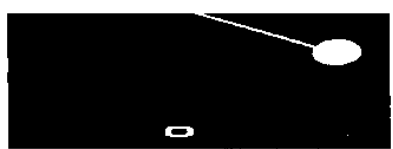

(a) Original even field

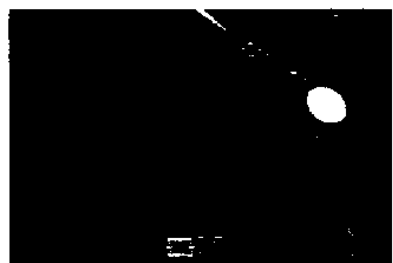

(c) Results by merging original even and odd field

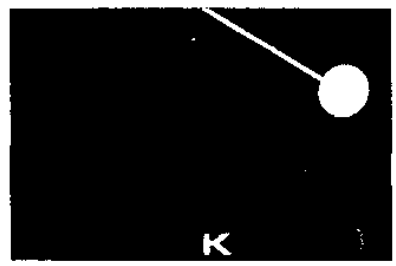

(e) Intra interpolation from original odd field

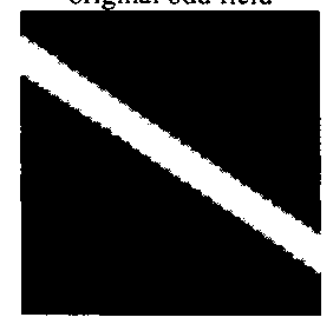

(g) Bilinear interpolation

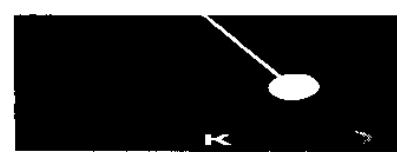

(b) Original odd field

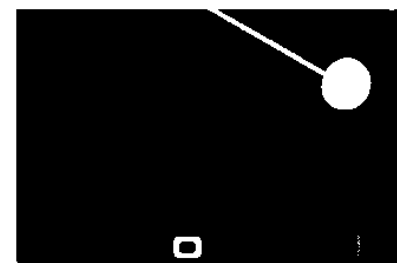

(d) Intra interpolation from original even field

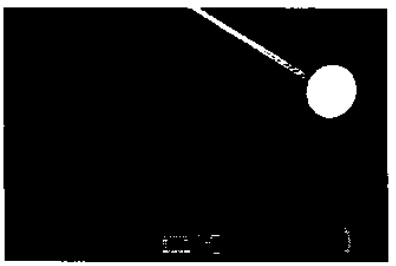

(f) Result by the 2-field motion detection

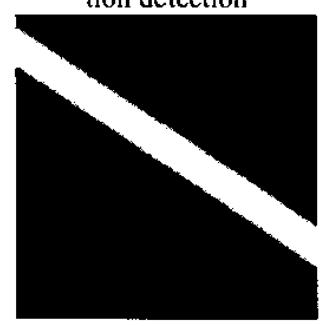

(h) Enhanced ELA

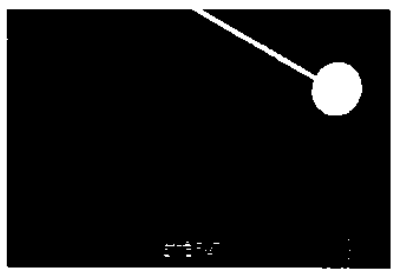

(i) Proposed method

Fig.6 The comparison of subject view
Edges in the picture could be recognized when intra-field deinterlacing is adopted. But the words " $\mathrm{O}$ " and " $\mathrm{K}$ " will disappear alternatively because they exist only in the different parity fields, shown as Fig. 6(d) and Fig. 6(e).

The resulting picture of the two-field motion detection [3] is shown in Fig. 6(f). Although the words "OK" are successfully preserved, the line crawling still exists in the string part of the pendulum.

For the intra-field de-interlacing, the bilinear interpolation and the ELA make differences with their sharpness. The result of the bilinear interpolation is shown as Fig. $6(\mathrm{~g})$, aliasing-edge can be seen. The result of the ELA is shown as Fig. 6(h), while the edgc is much sharper than the bilinear interpolation one.

The result of the proposed method which has 4-field horizontal motion detection and enhanced ELA is shown as Fig. 6(i). The edges of the pendulum and the logo are sharp, and the words "OK" are white words with black spaced in-between. The proposed method has overcome the tough hurdles of all the other deinterlacing methods.

\section{CONCLUSIONS}

A high quality, low cost and fast de-interlacing method is proposed in this paper. The ELA with Median processing keeps the sharp edge of the original field. The 4-field horizontal motion detection combines the techniques of the 4-field motion detection. The experiment results show that the proposed algorithm really has high picture quality, low memory access frequency and memory usage.

\section{REFERENCES}

[1] http://nickyguides digital-digest.com/index.htm

[2] Chung J. Kuo, Ching Liao, and Ching C. Lin, "Adaptive Interpolation Technique for Scanning Rate Conversion," IEEE Trans on Circuits and Systems for Video Technology, vol. 6, no.3, June 1996

[3] S.F. Lin, Y. L. Chang, and L. G. Chen, "Motion Adaptive Interpolation With Morphological Operation and 3:2 pulldowned Recovery for Deinterlacing", IEEE International Conference on Multimedia and Expo, Aug. 2002

[4] Kenju Sugiyama and Hiroya Nakamura, "A Method of Deinterlacing with Motion Compensated Interpolation," IEEE Trans Consumer Elec., vol. 45, no. 3, pp.611-616, August 1999.

[5] Kevin Hilman, Hyun Wook Park, and Yongmin Kim. "Using Motion-Compensated Frame-Rate Conversion for the Correction of 3:2 Pulldown Artifacts in Video Sequences," IEEE Trans. Circuits Syst. Video Technol., vol. 10 , no. 6, September 2000 .

[6] D. Van De Ville, B. Rogge, W. Philips, and I. Lemahieu, "Deinterlacing using fuzzy-based motion detection," Knowledge-Based Intelligent Information Engineering Systems, 1999. Third International Conference, 1999, pp263-267.

[7] A.J Patti, M.I Sezan and A.M Tekalp, "Robust Methods for High Quality Stills from Interlaced Video in the Prescnce of Dominant Motion," IEEE Trans. Circ. Syst. Video Tech., vol. 7, no. 2. pp. 328-342, Apr. 1997. 